\title{
DETERMINAÇÃO DO INTERVALO DE PÓS-MORTE DE CADÁVERES: IMPASSES ENCONTRADOS NAS TÉCNICAS UTILIZADAS PELA PERÍCIA FORENSE
}

\author{
Irineu Ferreira da Silva Neto ${ }^{1}$ \\ ${ }^{1}$ Curso de Bacharelado em Farmácia, Unidade Acadêmica de Saúde, Faculdade de Medicina \\ Estácio de Juazeiro do Norte, Juazeiro do Norte-CE, Brasil. \\ Email para correspondência: yrineuferreira@gmail.com
}

\begin{abstract}
Resumo
O Intervalo de Pós-Morte (IPM) é o período de tempo entre a morte e o momento em que o cadáver é encontrado, tendo grande importância nas circunstâncias do óbito e reconstituição de cenas. Objetivou-se descrever alguns dos principais impasses evidenciados nas técnicas utilizadas para determinação do IPM. Revisão de literatura com abordagem qualitativa nas bases de dados eletrônicas: SciELO, PubMed, e Google Scholar, de artigos publicados entre 2005 e 2019, por meio dos descritores: Antropologia Forense "Forensic Anthropology", Ciências Forenses "Forensic Sciences", Entomologia "Entomology", Prova Pericial "Expert Testimony" e Toxicologia Forense "Forensic Toxicology". Os estudos mostram as variadas técnicas para estimar o IPM, dentre elas: uso de insetos, arcada dentária, degradação óssea, sendo as mais descritas, cada uma com condições específicas, vantagens e desvantagens. Fatores ambientais, uso de algumas drogas, doenças degenerativas são fatores que podem interferir diretamente nos resultados e, consequentemente, induzir o erro. A partir da análise dos dados, pôde-se observar que existem algumas deficiências nas metodologias, e surge a necessidade de novos estudos experimentais para aprimorar as técnicas, auxiliando a perícia forense a desvendar os casos criminais.
\end{abstract}

Palavras-chave: Antropologia Forense, Ciências Forenses, Entomologia, Prova Pericial, Toxicologia Forenses.

\begin{abstract}
The Postmortem Interval (IPM) is the period of time between death and the moment when the corpse is found, having great importance in the circumstances of death and reconstitution of scenes. The objective was to describe some of the main impasses evidenced in the techniques used to determine the IPM. Literature review with a qualitative approach in the electronic databases: SciELO, PubMed, and Google Scholar, of articles published between 2005 and 2019, through the descriptors: Forensic Anthropology "Forensic Anthropology", Forensic Sciences "Forensic Sciences", Entomology "Entomology", Expert Testimony "Expert Testimony" and Forensic Toxicology "Forensic Toxicology". Studies show the various techniques for estimating IPM, among them: use of insects, dental arch, bone degradation, the most described, each with specific conditions, advantages
\end{abstract}


and disadvantages. Environmental factors, use of some drugs, degenerative diseases are factors that can directly interfere in the results and, consequently, induce the error. From the analysis of the data, it was possible to observe that there are some deficiencies in the methodologies, and there is a need for new experimental studies to improve the techniques, assisting the forensic expertise to unravel the criminal cases.

Keywords: Forensic Anthropology, Forensic Sciences, Entomology, Expert Evidence, Forensic Toxicology.

\section{Introdução}

As Ciências Forenses vêm a cada dia ganhando ênfase como forte e crucial elemento na investigação criminal, sendo fundamental para resolução de casos periciais. Essa área se correlaciona com a Genética, Entomologia, Botânica, Toxicologia, Antropologia, entre outras, para investigações legais mais precisas e contundentes, perante a criminalística, para que unidas desmembrem variados crimes (SANTOS, 2018). Nesse contexto, compreender o estado de decomposição de cadáveres é algo de suma importância para interpretação de casos forenses. É necessário também ter uma estimativa do Intervalo de PósMorte (IPM), dessa forma, se tem uma melhor aplicação da lei em questões jurídicas (FERREIRA; CUNHA, 2012).

O conceito de morte biológica está relacionado ao término de todos os processos fisiológicos e biológicos, por meio de diversas reações químicas, e dessa maneira, constitui uma irreversível perda da unidade que é o corpo humano. De tal forma, nasce a necessidade de definir o critério de morte e o período em que isso ocorreu para que se pronuncie como uma pessoa morta, podendo aplicar a legislação mais precisamente (GRIGULO, 2016).

O IPM pode ser definido como o período de tempo entre a morte e o momento em que o cadáver é encontrado, tendo grande importância nas circunstâncias do óbito, reconstituição de cenas, relação do suspeito com a cena do crime, e até mesmo em mortes de causas naturais, acidentais ou suicídios (ERMIDA, 2015). Para auxiliar na resolução de crimes, é relevante a incorporação de técnicas precisas, em especial, casos de crimes em que os tribunais provavelmente darão uma maior credibilidade a evidências biológicas devido a confiabilidade e segurança, comparado a provas oculares (SANTOS, 2018).

Após a morte, ocorre uma série de reações bioquímicas que trazem consigo inúmeras modificações estruturais e alterações na composição do 
corpo humano. Essas reações se manifestam de forma sequencial, e a partir dessa afirmação, tem sido estudado a utilização de técnicas que avaliam as diversas mudanças biológicas e o intervalo de tempo decorrido após a morte, já que esse processo, geralmente, ocorre de forma linear. No entanto, existem algumas limitações nas metodologias empregadas para determinar o IPM e cada vez mais estudos tem buscado melhorias neste sentido (FERREIRA; CUNHA, 2012; GARCIDUEÑAS et al., 2016; ANTONIO et al., 2018).

Dessa forma, objetivou-se descrever alguns dos principais impasses evidenciados nas técnicas utilizadas para determinação do IPM.

\section{Metodologia}

Trata-se de uma revisão de literatura, que tem como intuito abordar alguns impasses das técnicas utilizadas na perícia Forense e características encontradas na desenvoltura destas. Esse estudo de abordagem qualitativa foi realizado por meio das bases de dados eletrônicas: PubMed (National Library of Medicine), SciELO (Scientific Eletronic Library Online) e Google Scholar. Foram utilizados os Descritores em Ciências da Saúde (DeCS): Antropologia Forense "Forensic Anthropology", Ciências Forenses "Forensic Sciences", Entomologia "Entomology", Prova Pericial "Expert Testimony" e Toxicologia Forense "Forensic Toxicology". A pesquisa foi realizada no mês de março de 2020, concomitantemente, em todas as três bases de dados eletrônicas.

Esta produção científica utilizou estudos publicados entre 2005 e 2019, sendo eles de caratér exploratório, experimental ou descritivo, tendo estes grande importância para o embasamento científico desta revisão.

Como critérios de inclusão foram adotados artigos disponíveis na íntegra, publicados em periódicos nacionais ou internacionais nos idiomas português ou inglês, contendo no mínimo dois dos descritores já citados. Artigos em outros línguas, incompletos ou que não tinham relação com a temática, foram excluídos. Foram encontrados 375 artigos que abordavam sobre a temática. Mas, após a análise criteriosa dessa produção científica, restaram 10 artigos que deram origem a esta revisão de literatura. 


\section{$3 \quad$ Resultados e Discussão}

Há uma constante atualização da literatura com técnicas que visam a estimativa do IPM, estas contribuem significativamente para identificar mais precisamente e compreender da melhor forma a decomposição cadavérica em diferentes condições ambientais (FERREIRA; CUNHA, 2012).

No entanto, cada uma delas possuem suas limitações, o que, consequentemente, pode induzir a resultados errôneos. Assim, alguns autores já puderam evidenciar impasses encontrados nas técnicas utilizadas pela perícia forense para estimativa do IPM, conforme mostra o Quadro 1, onde pode-se observar as informações referentes a autoria/ano, título da pesquisa, país de publicação, bem como seus principais resultados.

Quadro 1. Caracterização da produção científica.

\begin{tabular}{|c|c|c|c|}
\hline AUTOR/ANO & $\begin{array}{l}\text { TÍTULO DO } \\
\text { ESTUDO }\end{array}$ & $\begin{array}{c}\text { PAÍS DE } \\
\text { PUBLICAÇÃO }\end{array}$ & $\begin{array}{c}\text { PRINCIPAIS } \\
\text { RESULTADOS }\end{array}$ \\
\hline $\begin{array}{c}\text { Megyesi; } \\
\text { Nawrocki; } \\
\text { Haskell, } 2005\end{array}$ & $\begin{array}{l}\text { Usando graus- } \\
\text { dias acumulados } \\
\text { para estimar o } \\
\text { intervalo pós- } \\
\text { morte de restos } \\
\text { mortais em } \\
\text { decomposição. }\end{array}$ & $\begin{array}{l}\text { Estados } \\
\text { Unidos }\end{array}$ & $\begin{array}{l}\text { Algumas técnicas } \\
\text { utilizadas pela péricia } \\
\text { forense possuem } \\
\text { limitações relacionadas a } \\
\text { temperaturas em que } \\
\text { encontram-se o cadáver, } \\
\text { assim, é importante } \\
\text { conhecer bem a } \\
\text { temperatura da superfície } \\
\text { ou solo as condições } \\
\text { específicas do local onde } \\
\text { o corpo é encontrado. }\end{array}$ \\
\hline Costa, 2007 & $\begin{array}{lr}\text { Entomologia } & \\
\text { Forense } & - \\
\text { quando } & \text { os } \\
\text { insetos } & \text { são } \\
\text { vestígios. } & \end{array}$ & Brasil & $\begin{array}{l}\text { Algumas metodologias } \\
\text { empregadas } \\
\text { estimativa do para } \\
\text { disponibilizam apenas de } \\
48 \text { horas após a morte } \\
\text { para ter maior eficiência } \\
\text { de exatidão. Porém, } \\
\text { muitas vezes, o corpo só } \\
\text { é encontrado em um } \\
\text { período de tempo maior. } \\
\text { Nesse contexto, surge a } \\
\text { necessidade rade } \\
\text { mecanismos de } \\
\text { investigativos } \\
\text { complexos e mais } \\
\text { informações adicionais } \\
\text { para melhor manejo dos }\end{array}$ \\
\hline
\end{tabular}




\begin{tabular}{|c|c|c|c|}
\hline & & & dados. \\
\hline $\begin{array}{l}\text { Vass, } \\
2011\end{array}$ & $\begin{array}{lr}\text { A } & \text { elusiva } \\
\text { fórmula } & \text { de } \\
\text { intervalo } & \text { post- } \\
\text { mortem } & \\
\text { universal. } & \end{array}$ & Suiça & $\begin{array}{l}\text { Os métodos com o } \\
\text { objetivo de estimar } \\
\text { especificamente o IPM } \\
\text { tem uma aplicabilidade } \\
\text { reduzida, essas ficam } \\
\text { comprometidas em sua } \\
\text { maioria por questões } \\
\text { ambientais. As fórmulas } \\
\text { de Vass (2011) apenas } \\
\text { podem ser aplicadas a } \\
\text { restos cadavéricos ainda } \\
\text { com tecidos moles, } \\
\text { dificultando a análise dos } \\
\text { restos esqueletizados, ou } \\
\text { seja, tem maior precisão } \\
\text { quando o IPM é pequeno. }\end{array}$ \\
\hline $\begin{array}{c}\text { Thyssen; } \\
\text { Grella, } 2011\end{array}$ & $\begin{array}{l}\text { Efeito da } \\
\text { escopolamina } \\
\text { sobre o } \\
\text { desenvolvimento } \\
\text { de Chrysomya } \\
\text { putoria (Diptera: } \\
\text { Calliphoridae) e } \\
\text { sua importância } \\
\text { para a } \\
\text { estimativa do } \\
\text { intervalo pós- } \\
\text { morte. }\end{array}$ & Brasil & $\begin{array}{l}\text { Algumas substâncias } \\
\text { químicas encontradas nos } \\
\text { cadáveres podem afetar o } \\
\text { desenvolvimento ou inibir } \\
\text { as larvas de moscas } \\
\text { necrófagas, } \\
\text { comprometendo a análise } \\
\text { da entomologia, dentre } \\
\text { elas: benzodiazepínicos, } \\
\text { analgésicos, } \\
\text { anfetamínicos, heroína e } \\
\text { cocaína. Dessa forma, } \\
\text { pode-se levar } \\
\text { subestimativa errônea do } \\
\text { tempo de morte, tendo em } \\
\text { vista que os IPM são } \\
\text { calculados na entomologia } \\
\text { levando em consideração } \\
\text { o peso e idade da larvas } \\
\text { coletados do corpo. }\end{array}$ \\
\hline $\begin{array}{c}\text { Foltran; } \\
\text { Shibatta, } 2011\end{array}$ & $\begin{array}{l}\text { A ciência } \\
\text { forense e as } \\
\text { principais áreas } \\
\text { auxiliares. }\end{array}$ & Brasil & $\begin{array}{l}\text { É necessário que o exame } \\
\text { pericial para estimativa do } \\
\text { IPM seja composto de } \\
\text { uma vasta gama de } \\
\text { vestígios e técnicas em } \\
\text { áreas distintas, pois é } \\
\text { uma ciência } \\
\text { multidisciplinar sendo } \\
\text { crucial a união destas } \\
\text { unidades, pois só partindo } \\
\text { desse princípio é feita } \\
\text { uma análise correta, uma } \\
\text { vez que utilizando }\end{array}$ \\
\hline
\end{tabular}




\begin{tabular}{|c|c|c|c|}
\hline & & & $\begin{array}{l}\text { técnicas combinadas pode } \\
\text { haver maior precisão nos } \\
\text { resultados. }\end{array}$ \\
\hline $\begin{array}{c}\text { Ferreira; } \\
\text { Cunha, } 2012\end{array}$ & 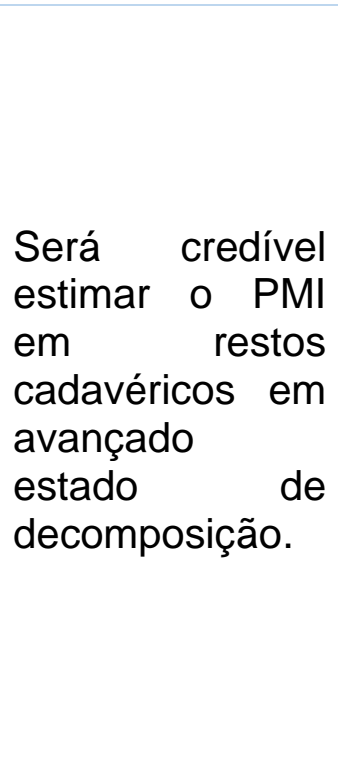 & Brasil & $\begin{array}{l}\text { É necessário ainda uma } \\
\text { maior transparência dos } \\
\text { processos de } \\
\text { decomposição em } \\
\text { humanos, já que a maioria } \\
\text { dos estudos } \\
\text { desenvolvidos até o } \\
\text { presente momento são } \\
\text { baseados em métodos } \\
\text { que tiveram os animais } \\
\text { como fonte de estudo. } \\
\text { Além disso, é essencial } \\
\text { buscar metodologias que } \\
\text { possam ser aplicadas } \\
\text { mais precisamente para } \\
\text { cadáveres humanos. }\end{array}$ \\
\hline $\begin{array}{c}\text { Pinheiro et al., } \\
2012\end{array}$ & $\begin{array}{lr}\text { Variáveis } & \text { na } \\
\text { estimativa } & \text { do } \\
\text { intervalo } & \text { pós- } \\
\text { morte } & \text { por } \\
\text { métodos } & \text { de } \\
\text { entomologia } & \\
\text { forense. } & \end{array}$ & Brasil & $\begin{array}{l}\text { Existem variáveis que } \\
\text { afetam direta } \\
\text { indiretamente } \\
\text { estimativa do IPM, } \\
\text { dificultando a precisão das } \\
\text { técnicas em vigor. São } \\
\text { fatores cruciais e } \\
\text { influentes: as condições } \\
\text { climáticas, local, tamanho } \\
\text { do corpo, agentes tóxicos, } \\
\text { vestimentas, ferimentos, } \\
\text { soterramentos, } \\
\text { comportamento noturno, } \\
\text { predatismo e outros. }\end{array}$ \\
\hline $\begin{array}{l}\text { Fruehwirth; } \\
\text { Folha, } 2017\end{array}$ & $\begin{array}{l}\text { Simulação do } \\
\text { uso da } \\
\text { entomologia } \\
\text { para estimar o } \\
\text { intervalo pós- } \\
\text { morte. }\end{array}$ & Brasil & $\begin{array}{l}\text { Existem objeções em } \\
\text { técnicas entomológicas } \\
\text { por não ser condizente } \\
\text { com o tempo real de } \\
\text { exposição, ou seja, o } \\
\text { cálculo de DGA (grau-dia } \\
\text { acumulado) tem } \\
\text { efetividade comprometida, } \\
\text { embora talvez seja porque } \\
\text { essa técnica baseia-se no } \\
\text { desenvolvimento da larvas } \\
\text { e os estudos } \\
\text { experimentais ocorrem, } \\
\text { geralmente, em } \\
\text { temperatura e umidade } \\
\text { diferentes ao local do } \\
\text { crime, e esse fato pode }\end{array}$ \\
\hline
\end{tabular}




\begin{tabular}{|c|c|c|c|}
\hline & & & $\begin{array}{l}\text { justificar os resultados } \\
\text { divergentes encontrados. }\end{array}$ \\
\hline $\begin{array}{c}\text { Pereira; } \\
\text { Canettieri, } \\
2017\end{array}$ & $\begin{array}{l}\text { Identificação } \\
\text { post-mortem } \\
\text { baseada na } \\
\text { análise da } \\
\text { arcada dental: } \\
\text { Relato de caso. }\end{array}$ & Brasil & $\begin{array}{l}\text { Para } \\
\text { carbonizadas, existem } \\
\text { métodos de identificação } \\
\text { do IPM através da } \\
\text { avaliação da arcada } \\
\text { dentária, com o objteivo } \\
\text { de comparar com o } \\
\text { prontuário odontológico do } \\
\text { paciente, sendo de baixo } \\
\text { custo, ágil e confiável. } \\
\text { Porém, esse } \\
\text { procedimento só é } \\
\text { possível quando se possui } \\
\text { o prontuário do indivíduo, } \\
\text { e deve-se levar em } \\
\text { considerações nas novas } \\
\text { metodologias as pessoas } \\
\text { indigentes e outros que } \\
\text { não detém } \\
\text { documentação. }\end{array}$ \\
\hline $\begin{array}{l}\text { Correia, } \\
2019\end{array}$ & $\begin{array}{l}\text { Análise do } \\
\text { acervo ósseo do } \\
\text { sítio Largo da } \\
\text { Matriz, Marechal } \\
\text { Deodoro - AL: } \\
\text { das patologias } \\
\text { às fraturas. }\end{array}$ & Brasil & $\begin{array}{l}\text { A análise da arcada } \\
\text { dentária e óssea pode } \\
\text { relevar inúmeras } \\
\text { informações relacionadas } \\
\text { a relação saúde/doença } \\
\text { do indivíduo. No entanto, } \\
\text { para empregar este tipo } \\
\text { de metodologia, deve-se } \\
\text { levar em consideração } \\
\text { também processos } \\
\text { degenerativos, infecciosos } \\
\text { nos ossos, algumas } \\
\text { patologias e desgastes } \\
\text { dentários, pois esses } \\
\text { fatores podem } \\
\text { comprometer o tecido } \\
\text { ósseo a } \\
\text { consequentemente, a } \\
\text { análise. }\end{array}$ \\
\hline
\end{tabular}

Fonte: Dados da pesquisa, 2020.

Indentifica-se que no recorte temporal delimitado pelo estudo houve uma publicação referente a temática estudada em 2005; uma em 2007; três em 2011; duas em 2012; duas em 2017 e uma em 2019. A maioria dos estudos expõe que os fatores e condições ambientais estão atrelados aos resultados das estimativas do IPM. 
No Quadro 2 pode-se observar as principais limitações evidenciadas a partir do levantamento realizado na literatura.

Quadro 2. Fatores limitantes das técnicas utilizadas pela perícia forense.

PRINCIPAIS FATORES LIMITANTES

Rápida decomposição cadáverica;

Comprometimento da análise devido a presença de drogas; A maioria dos estudos, até 0 momento, foram realizados apenas em animais.

Doenças degenerativas e infecciosas; Impossibilidade de haver o prontuário ondontológico do paciente.

Temperatura da superfície e condições ambientais; Aplicação de apenas uma metodologia para estimativa; Interferência de predatismo animal.
ÁREA PERICIAL

Entomologia forense

Fonte: Dados da pesquisa, 2020

Compreensivelmente, o consenso geral recomenda a realização de qualquer avaliação pós-morte o mais rápido possível após a morte, uma vez obtido o consentimento da família. No entanto, esses prazos curtos podem dificultar a viabilidade do procedimento (HURTADO et al., 2018).

Segundo Costa (2011) as estimativas de pós morte podem ser realizadas em 5 estágios, esses foram descritos como: Evaporação Tegumentar; Rigidez Cadavérica; Resfriamento do Corpo; Livores Cadavéricos e Autólise ou Putrefação. Esses são momentos em que é possível fazer avaliação do período de pós morte a curto prazo. Metodologias a longo prazo mais eficientes, precisas e de baixo custo ainda são necessárias para que possam ser aplicadas na perícia forense.

Em geral, a presença de organismos específicos pode constituir evidência, auxiliando na determinação da causa de determinada morte, atuando como bioindicadores de processos criminais, como é estudado na entomologia forense. Mas, como já foi evidenciado, essa área possui deficiências, especialmente relacionadas a fatores ambientais (OLIVEIRA; AMORIM, 2018).

O estudo de Foltran; Shibatta (2011) destaca que algumas das metodologias já existentes não são fidedignas ao serem avaliadas 
isoladamente para as estimativas dos IPM, sendo necessário metodologias combinadas para entender o processo mais precisamente.

Sendo assim, os métodos que interferem as estimativas de IPM são difíceis de serem estabelecidos, pois são suscetíveis a muitos fatores externos e ambientais, como temperatura, umidade, tensão de oxigênio, insetos e atividade necrófaga (DONG et al., 2019).

Ferreira; Cunha (2012) relatam que pode ocorrer variabilidade no tempo de decomposição em indivíduos que possuem o mesmo período de pós morte, sendo assim, as estimativas devem levar em considerações as condições específicas de cada situação. E, no caso da antropologia forense, há uma árdua tarefa de expressar o período de pós-morte apenas com escassas peças ósseas, muitas vezes são utilizadas o crânio, pois ele tem uma durabilidade maior, comparado aos outross ossos do corpo humano. Por meio da análise de partes dos ossos busca-se a estimativa de IPM mais credível, apesar de haver uma facilidade muito maior quando se está presente o corpo completo.

O tecido ósseo tem grande resistência a ações do meio externo, desse modo, é também conhecido por ser um local de grande importância para observações idiossincráticas, por isso, o potencial de algumas estruturas ósseas é enaltecido em algumas métodos da Antropologia Forense (PEREIRA, 2018).

O princípio da perenidade pode ser descrito por meio de elementos de corpo humanos terem grande dificuldade para sofrerem alterações, geralmente, os ossos ao resistirem à ação do tempo, mantendo-se em grande conformidade por um período prologado após a morte, por esse e outros motivos a análise de ossos é muito utilizada desde a antiguidade (SCHEMES, 2014).

Dessa forma, pelo que já se pode concluir pela paleontologia, a duração máxima de ossos a superfície é de 20 anos. Ou seja, já que o último tecido a se desintegrar é o tecido ósseo, os IPM que ultrapassarem esse período tem uma chance muito pequena de serem revelados (CORREIA, 2019). 


\section{Conclusão}

A partir da análise, constata-se que existem variadas técnicas para estimar o IPM de cadáveres, sendo elas: graus-dias acumulados; insetos como vestígios; análise da árcada dentária; determinação do acervo ósseo e avaliação dos restos cadavéricos, cada uma com suas especificações próprias.

Dentre as limitações encontradas na literatura, destacam-se: Rápida decomposição cadáverica; Comprometimento da análise devido a presença de drogas; Poucos estudos e realizados apenas em animais; Doenças degenerativas e infecciosas; Impossibilidade de haver 0 prontuário ondontológico do paciente; Temperatura da superfície e condições ambientais; Aplicação de apenas uma metodologia para estimativa e interferência de predatismo animal. Mas, apesar de haver atualizações recorrentes sobre as técnicas supracitadas, ainda é essencial um aprimoramento destas.

É necessário técnicas que sejam precisas, de baixo custo e que possa ser aplicadas em diversas situações, esse estudo sugere o aperfeiçoamento de metodologias por meio de estudos experimentais. Os requisitos citados, anteriormente, são essenciais para ajudar a ciências Forenses a desvendar e refazer provas circunstâncias que são de suma importância para resolução dos casos criminais.

\section{Referências}

ANTONIO, A. S. et al. Avaliação de características químicas do solo durante a decomposição cadavérica e suas aplicações à química forense. Revista Virtual de Química, v. 10, n. 3, 2018.

CORREIA, S. J. Análise do acervo ósseo do sítio Largo da Matriz, Marechal Deodoro - AL: das patologias às fraturas. 2019. $48 \mathrm{f}$. Trabalho de Conclusão de Curso (Graduação em Arqueologia) - Campus de Laranjeiras, Universidade Federal de Sergipe, Laranjeiras, 2019.

COSTA, J O. Entomologia Forense - quando os insetos são vestígios. Millennium, v. 2, p. 167-264, 2007. 
COSTA, J O. Entomologia Forense, quando os insetos são vestígios. Tratado de Perícias Criminalísticas. Millennium. Campinas, Brazil, v. 3, p. 502, 2011.

DONG, K. et al. Succession of oral microbiota community as a tool to estimate postmortem interval. Scientific reports, v. 9, n. 1, p. 1-9, 2019.

ERMIDA, A. C. M. Luminol: contributo para a estimativa do intervalo postmortem e de restos estilizados. 2015. 121 f. Tese (Mestrado em Medicina Legal e Ciências Forenses) - Universidade de Coimbra, Combra, 2015.

FERREIRA, M. T.; CUNHA, E. Será credível estimar o PMI em restos cadavéricos em avançado estado de decomposição. Cadernos do GEEvH, v. 2, p. 7-20, 2012.

FRUEHWIRTH, M.; FOLHA, R A.. Simulação do uso da entomologia para estimar o intervalo pós-morte. Colloquium Vitae, v. 9, p. 53-58, 2017.

FOLTRAN, R. K.; SHIBATTA, L. A ciência forense e as principais áreas auxiliares. Atenção ao idoso ação multiprofissional em saúde, v. 1, p. 15-16, 2011.

GARCIDUEÑAS, A. L. C. et al. Forensic study of skin post-mortem changes as a supplementary test to determine post-mortem interval (first 78 hours). Colombia Forense, v. 3, n. 2, p. 27-33, 2016.

GRIGULO, M. M. M. Entomologia forense: os insetos de maior importância para a ciência criminal. Jornada Integrada em Biologia, p. 47-58, 2016.

HURTADO, J. C. et al. Postmortem interval and diagnostic performance of the autopsy methods. Scientific reports, v. 8, n. 1, p. 1-10, 2018.

MEGYESI, M. S.; NAWROCKI, S. P.; HASKELL, N. H. Using accumulated degreedays to estimate the postmortem interval from decomposed human remains. Journal of Forensic Science, v. 50, n. 3, p. 1-9, 2005.

OLIVEIRA, M.; AMORIM, A. Microbial forensics: new breakthroughs and future prospects. Applied microbiology and biotechnology, v. 102, n. 24, p. 10377-10391, 2018. 
PEREIRA, N. O.; CANETTIERI, A. C. V. Identificação post-mortem baseada na análise da arcada dental: Relato de caso. Revista Univap, v. 22, n. 40, p. 676, 2017.

PEREIRA, M. F. G. Identificação Humana através das particularidades ósseas no exame radiográfico da coluna vertebral e tórax. 2018. 166 f. Dissertação (Mestrado em Medicina Legal) - Universidade do Porto, Porto, 2018.

PINHEIRO, D. S. et al. Variáveis na estimativa do intervalo pós-morte por métodos de entomologia forense. Enciclopédia Biosf, Goiânia, v. 8, p. 1442-1458, 2012.

SANTOS, A. E. As principais linhas da biologia forense e como auxiliam na resolução de crimes. Revista Brasileira de Criminalística, v. 7, n. 3, p. 12-20, 2018.

SCHEMES, B. I. A. Importância da antropologia forense na responsabilização dos crimes contra a humanidade praticados no Brasil em regimes autoritários. Rev. Fac. Direito Saeo Bernardo do Campo, n. 20, 2014.

THYSSEN, P. J.; GRELLA, M. D. Efeito da escopolamina sobre o desenvolvimento de Chrysomya putoria (Diptera: Calliphoridae) e sua importância para a estimativa do intervalo pós-morte. Revista Brasileira de Criminalística, v. 1, n. 1, p. 39-42, 2011.

VASS, A. A. The elusive universal post-mortem interval formula. Forensic science international, v. 204, n. 1-3, p. 34-40, 2011. 